\title{
Ephemeral Social Media Visuals and Their Picturesque Design: Interaction and User Experience in Instagram Stories
}

\author{
Balca Arda \\ Kadir Has University (Istanbul, Turkey) \\ E-mail: balca.arda@khas.edu.tr
}

\begin{abstract}
This article examines the temporality of ephemeral visual posts to social media with an emphasis on Instagram stories. Drawing on theories of the spectacle, it is my contention that interaction and user-experience design, as it pertains to social media platforms, highlights the contemporary conditions and motivations in our society of abundant visual consumption. This article investigates what it means for a social media user to attend to such time-related visual experience. Throughout this piece, I critically survey the relationship between ephemeral Instagram stories' popularity and the high speed temporality of the social media sphere with emphasis on the digital "picturesque." Perishable daily sharing on social media reflects a contemplative glimpse into a personal lifespan presented as an object of mass appreciation. I examine how ephemerality as a component of design impacts online sociality through the picturesque visual mode. Contemporary boredom and competitive engagement in high-technological communication networks inform how we might direct digital publics to find alternative pathways to sociality.
\end{abstract}

Keywords: picturesque, contemplation, contemporary boredom, Instagram, temporality.

\section{Introduction}

Monthly active users of social media are expected to exceed 3 billion people in the year 2021 (Chen and Cheung 2019). Facebook, YouTube, Twitter, WhatsApp, and Instagram are among the leading social media platforms worldwide (Clement 2020b). These online platforms enable "persistent channels of mass-personal communication" and they facilitate the "perceptions of interactions among users" and derive "value primarily from user-generated content" (Carr and Hayes 2015, 49). Social media as a medium reclaims "a chaotic atemporality mixing the past 
with present and the future" (Jurgenson 2013a) while allowing a permanent archival capacity and instant chatting opportunities based in "real time." Social media seems to put an emphasis on "an assumed inevitability of recording most everything forever” (Jurgenson 2013a). Yet recent developments to various social media platforms including Snapchat and Instagram have also facilitated ephemeral recordings in addition to the permanent and enduring posts that we have become so used to. These sites "allow an individual user to share content for a limited time with auto-deletion” (Khalil and Najaf, 2019), aka temporal sharing. This ephemeral content includes Snaps or My Story in Snapchat, Instagram Stories, YouTube Stories, as well as Facebook statuses and WhatsApp Statuses. Temporal visual posts are less filtered than the usual newsfeed and assumingly reflect an authentic claim besides their focus on the presence of the present instead of memory captions.

Time is an inherent factor in social media use. Duration is a specific component of visual posts for users of social media. In this article, I will explore the temporal design structure of ephemeral visual posts and their spectacle traits by examining viewer attentiveness. I argue that ubiquitous production in social media also provokes competition to attract and hold viewers' attention. Indeed, social media ensures a peculiar contemplation time to viewers. Boris Groys has named this contemplation in the social media sphere as "cool contemplation" (Groys 2011)." Such contemplation defines active engagement that paradoxically does not evolve into a profound judgmental process and comprehension. Therefore, social media use entails looking but not seeing in a genuine sense.

Yet, different kinds of interaction designs can motivate alternative approaches to contemplate and interact with the posts that have been shared. The stillness of perishing visual posts imposes a different standard of appreciation in conjunction with modern boredom that Martin Heidegger connects with the drive for technological change (Heidegger 1996). Social media is known to be a pass-time activity that connotes with escapism from daily life. The picturesque mode of seeing is escapist as is the safety found in the pastoral scene, while dominant power, aka the city awaits there beyond the hill (Robinson 1988, 75). As such, the picturesque landscape "draws us in" to a retreat enabling "a broader, safer perspective, an aestheticizing distance" (Mitchell 2002, viii). Social media engagement has been generically correlated with mediated voyeurism. Visual posts capturing individual lives can be visualized by the followers of the user's account through the platform. This mediated voyeurism creates companions in a daily life made up of ubiquitous connectivity. But more importantly, the visuality of social media provides refuge as a way of "filling in time" (Hand 2016, 117). Throughout this article I will focus on Instagram stories and 
the ways these stories mediate in picturesque manner. Ephemerality has become a central part of social media and the public sphere. Each portion of shared content has an exhibition time - it is available for up to 24 hours, while photos and videos disappear after a limited time interval, variable among platforms. I aim to provide a theoretical framework of the picturesque as it is found in social media in order to better understand our historical practice of both exhibiting and viewing. In doing so, I undertake Instagram as a tool of the spectacle in relation to visual aesthetics that arranges the way the user senses and experiences the sensible. As such, I explore how the interaction design of time-related visual posts confronts the scarce attention span of the modern human and contemporary boredom.

\section{Theoretical Methodology}

The format for design of user-experience and interaction in social media vis-àvis contemplative attitude is relatively under-analysed. I analyse this peculiar contemplation mode in digital public sphere through temporal subscripted structure of such visual posts. As methodological framework, this article adopts a theoretical analysis of the visual spectacle through the time-sensitive interaction of Instagram stories. I explore ephemeral media use as practice while focusing on the design structure of "visual engagement" (Kress and van Leeuwen 1996). The structure design of interaction and user experience provides information on how the spectacle of social media has been envisaged to proceed as such "the ability of an image to attract, involve and engage with users" (Valentini et al. 2018, 365). In order to accomplish this, I will construct my theoretical study drawing on previous qualitative and quantitative research on ephemeral social media interaction and user experience projections. The visuals mentioned in this article do not form a representative sample, yet they exemplify picturesque applications of ephemeral social media. I analyse the interaction design centred user experience of Instagram as it causally mediates between the participants' world, which is represented and the audience's world, where representation is consumed. This article does not seek to explain what exactly happens when one consumes ephemeral social media content but aims instead to explore the structure of interaction and user experience design. Deriving from practice theory, I acknowledge social media posts/receipts "are necessary elements and qualities of a practice in which the single individual participates, not qualities of the individual” (Reckwitz 2002, 250). Indeed, the time dynamics for the production and consumption of social media posts corresponds to how power works in the broader media sphere. I contend that the choice for interaction 
and user experience design for social media gives us clues about the conditions and motivations of our speedy society of high-technological communication.

\section{Instagram: An App for "Cool” Contemplation}

Contemporary image culture is defined by communication technologies that provide ways for subjects to engage with "systems of conventions and techniques such as narrating, editing, composing, lightning, sequencing” (Manovich, 2016, 18) and other visual traits and qualities. Instagram provides a digital space for users to engage with visual creativity. At the same time it "maintains and cultivates existing relationships as a social network site” (Sheldon 2008; Sheldon et al. 2017, 644). Instagram, among other social media platforms, offers specifically image related posts that prioritize the users' own performance and appreciation of visual aesthetics to facilitate their involvement in digital publics.

Instagram was launched as a photo-sharing platform in 2010 and bought by Facebook company in 2012. The Instagram app, one of the most popular social networking apps worldwide, permits its users to edit and share self-authored still and moving images. What makes Instagram unique is that Instagram presents a more polished, aesthetically driven, and hence well thought-out sharing practice in comparison to Snapchat (Kofoed and Larsen 2016). Leo Manovich (2016) asserts that Instagram's image culture reflects "a rich cultural and historical context, including histories of photography, cinema, graphic design, as well as contemporary social media, design trends, music video, and k-pop," while its users, a “young global generation are connected by common social media platforms, cultural sensibilities, and visual aesthetics" (Manovich 2016, 4).

Contemporary digital contemplation necessarily distinguishes itself through the specific design of interaction and participant experiences. Instagram offers several different ways of forming and engaging with DIY visual media spectacle for its users. Instagram is primarily a mobile-only image taking, editing and sharing application. Instagram users can see their feed, profiles, and comments via desktop, but they cannot post without mobile phones. The posts in the newsfeed are automatically stored in the profile account of the poster. Instagram stories which are temporary content do not appear in the user's feed and are not visible in the poster's profile account (Javed 2019). However, a temporary Instagram story can also be stored in the poster's profile as highlights, which are stored permanently and will not vanish after 24 hours, but this does not happen automatically as with other posts. Followers cannot duplicate a post running in the newsfeed or Instagram story unless an 
exterior application intervenes to allow such features. Instagram conjugates with the conceptualization of networked publics that primarily enable public communication amongst followers in contrast to Snapchat, which prioritizes private mediation.

Indeed, time scarcity and the overpopulation of information in social media means that not every post is guaranteed to be thought through in-depth or even seen in the newsfeed. An ideal interaction design to be used for the digital public would eventually transform the current sociality precepts "from its current state to a preferred state" (Zimmerman, Forlizzi and Evenson 2007, 493). Contemplation, in a traditional sense, signifies thoughtful observation and meditation. According to Groys (2011), "cool" contemplation derives from the lack of time to engage with a comprehensive account of the object of appreciation. The central question is how a single visual post can become contemplative and if so what kind of contemplation design it is. Interaction design aims to provide an enjoyable user experience. As such a user-centred design process for an application involves "considering who is going to use the interactive products, how they are going to use them and where they are going to be used" (Preece, Sharp and Rogers 2015, sec. I). As Manovich explains, the Instagram user frequently expresses a particular sensibility of "being in the scene pathos" (Manovich 2016,135 ) as well as a communicative mood established in the way that s/he arranges the posts (Manovich 2016, 77): "personal, emotional and moody" (Manovich 2016, 106). Thus, this study attends to design exploration (Fallman 2008, 7-8) in order to contribute to current societal needs of communication.

\section{Experiencing Time through Social Media in a High- Speed Society}

When time is at a scarcity, social media posts appear as a symptom of boredom. The precarity conditioned by contemporary capitalism indicates not only the instability of working conditions but also the scarcity of time. We do not have much time to attend to anything in a comprehensible manner in our speedy daily lives. PEW research states that home usage is ubiquitous among smartphone owners and that both young and old are similarly prone to using their phones while in a car or on public transit ( $85 \%$ of younger users and $79 \%$ of older users did so), as well as in a community space such as a park or coffee shop (Smith 2015). Our leftover time, such as the time spent during daily commutes is mostly consumed by social media activation. As Susan J. Matt articulates in her interview with Sean Illing; smartphone applications, including social media, promise "constant companionship, fulfilment and excitement, instantaneous entertainment and variety" (Illing 2019). 
Visually abundant social media has become the new agora for an international public at the edge of the dilution between the public and private sphere, where the boundaries between work-, home- and leisure-time have disappeared. Among the motivations to use social media, scholars have identified "social interaction, information seeking, passing time, entertainment, relaxation, communicatory utility, convenience utility, expression of opinion, information sharing and surveillance and knowledge of others" (Alhabash and Ma 2017, 4; Whiting and Williams 2013). For many, social media provides something to do to pass a given imposed duration of time. One of the primary motives for using Instagram is escapism along with social interaction, archiving, selfexpression and peeking. Here, escapism includes "to escape reality," "to forget about troubles," "to avoid loneliness," "to get what I want without much effort," and "to relax" (Lee et al. 2015, 554). The mismatch between the subject's time; the user of social media, and object's time; bus travel duration, has been nullified. The user cannot control the commuting speed yet can rule the newsfeed scrolling tool.

A vast number of social media users have easily become objects of surveillance, attention, and contemplation. As Groys suggests, more people are interested in image production today than in image contemplation (Groys 2011, 15). He goes on to argue that the "easy access to digital photo and video cameras combined with the global distribution platform of the internet has altered the traditional statistical relationship between image producers and image consumers" (Groys 2011, 12-15). Sheldon and Bryant contend that Instagram users focus more on personal identity and self-promotion as well as surveillance in the sense of knowledge gathering about others and documentation of life events in addition to displaying creativity such as photographic skills rather than connection with other people (Sheldon and Bryant 2016). Thus, surveillance is the main motivation for Instagram usage (Alhabash and Ma 2017). Shared photos are used firstly to talk about images as conversational resources, to communicate visually with a focus on visual modalities, and phatic interaction between users for the sake of visual connectivity to confirm and strengthen relationships (Kofoed and Larsen 2016).

Social media pass-time activities prevent the intervention of boredom. However, in addition to be "bored by something," Heidegger (1962) introduces the notion of profound boredom as becoming bored with. This profound boredom does not proceed in relation to specific objects or events (Heidegger 1962). For instance, one can become "bored by downloading speed" and then engage with social media in the meantime. But profound boredom designates the state where nothing any longer appeals. "A positive refusal that presents an emptiness by which we do not expect anything from our surroundings, by which the world has fallen dead" 
(Hammer 2004, 282). Thus, although social media can be diverting, it also belongs to an 'information overload' conditioned by the omnipresence of technology. Virilio attributed capitalist workloads with expectations of constant communication. Speed addresses a paradoxical phenomenon in which the technological development that enables the greatest extension of mass participation also magnifies control over the public of the high-speed society (Virilio 2005). Virilio asserts that this neverending instantaneous communication is arrhythmic and because of that it terrifies the habitants of the accelerated reality (Virilio 2012, 27). Constant anxiety rises from stimulation of this threshold. Thus, social media can provoke indifference for the user who is bored of society's unsatisfiable high-speed demands. Indeed, users who are scrolling social media newsfeeds fulfil the daily expectations of a constant connective mode. Social media engagement is part of our contemporary temporal routine. When multitasking and constant immediacy are the requirement, "the time we're allowed to concentrate exclusively on one thing is progressively diminishing" (Rosa and Scheuerman 2009, 1-2). This is because we are constantly being interrupted by a stream of incoming news and messages. The habitant of this high-speed society notices that $s /$ he is not in charge of time but rather subject to time and even kept in limbo. Thereby, social media use can also provoke a profound boredom, a kind of acknowledged boredom, for its wanderers. Contemporary highspeed society does not experience time. Neither work nor home nor leisure can make one experience time. According to Heidegger, to find something to do in order to pass time does not mean that time passes any more quickly, but rather that it becomes completely annulled and thereby one does not notice time passing (Heidegger 1996, 99; Misek 2010, 779). Thus, as Michael Hand (2016) puts forward, such focus on "now" and the constant movement among distractive tasks and events do not allow us to experience an authentic connectedness through time, namely the Heideggerian "presence of the present" (Heidegger, 1996). In the same vein, Kracauer associates the lack of boredom with absence from ourselves (Kracauer 1995, 331-334). Following this path, if boredom is being alone with oneself then it is authentic as it appeals to "the conscious self-coming to terms with Being in the World, and within one's own experience" (Ladly n.d., 145). iTime designates the user's monotonous, passive relationship to a culture of "speed and immediacy" and fractured relationships (Hand 2016, 117). This designates a kind of homesickness in the sense of being exiled. It follows that boredom is an unacknowledged homesickness that constitutes the perquisite of philosophy (Hammer 2004, 282).

There is a decrease in time devoted to image contemplation in the social media spectacle, where we experience a bombardment of posts. Daily social media use is 
part of our communal activities but even in archival mode posts to social media can remain unnoticed by the audience, who are simultaneously facing shortages of time and attention span. Consequently, the visuals posted to social media do not correspond to genuine contemplation. Posts to the newsfeed roll rather than temporal stories seem to be more inviting of contemplation because the spectator has more time to concentrate on the post if she/he desires. However, the aesthetic component of Instagram posts must compete with the networks' features because a photo can disappear down the stream, largely unnoticed (Jurgenson 2013b). Such unpopular posts, although archived on the poster's profile account, would be unlikely to be absorbed by the commonsensical historical process. Although liked, or commented on, or stored by captioning such active engagement with visuals in social media does not guarantee acknowledgement. As Groys explains, the "cool contemplation" of time-based media simply consists in "the permanent repetition of the gesture of looking, an awareness of the lack of time necessary to make an informed judgment through comprehensive contemplation" (Groys 2011, 100). Thereby, time-based contemporary art presents for the viewer "wasted excessive time that cannot be absorbed by the spectator" while it erases the difference between vita active and vita contemplative (Groys 2011, 100). Here, social media use as a contemporary performative act designates a kind of cool contemplation as users engage in active participation via the internet. Yet, such active engagement does not extend to generating understanding although it does not define passive spectatorship. As such, profound boredom emerges by acknowledging the inauthenticity of the social media roll as a worldly, everyday life experience, aka the loss of Being-there (Dasein) (Ladly n.d., 145-148).

Social media newsfeed, temporal posts like Instagram Stories deserve a closer examination of their role in adding value to the image in the era of increased visual abundance (Duin 2018, 11). Launching in August 2016 after 6 years of its release as an app in 2010, Instagram stories gathered 500 million daily active users (Clement 2020a). Stories run at the top part of users' Instagram newsfeed. When clicked on, Instagram stories appear on the full screen for 15 seconds. Here, users can move back and forth to previous and following stories in chronological order. Users can also stop the running of stories through by holding their finger on the image. The succession of stories does not need the viewer's command. This is in contrast to the newsfeed, where the viewer can watch stories without an extra move including interaction. These temporal stories combine videos and moving image features with photos of "still images and create a slideshow gallery" (Khalil and Najaf 2019, 163) that constructs an Instagram profile story. The temporality of ephemeral media is 
used to visually exhibit the self to the viewing followers. Ephemeral stories intensify our relationship with the self while they are at the same time perishable, and thus, non-static content. In a sense, such posts aim to compensate for fading subjectivity in a high-speed society.

\section{The Picturesque in the Ephemeral Visuality of Social Media}

Social media entertains through diversion and thus does not provide a view from a distance. The social media spectacle as a prevention against boredom, a visual passtime activity seemingly contrasts with the picturesque mode of seeing. Picturesque aesthetics have been generically connected to nature, natural settings and object in the sense that 'the term 'picturesque' was often but not always applied to books of landscape views" (Helsinger 2002, 120). The picturesque seemingly provides a safety found in the pastoral scene, a break from the unnaturalness and extravagance of the court (Robinson 1988, 75). Yet, ephemeral visual posts on social media project another, nevertheless parallel kind of spectacle. The temporal features provide a pause so that the spectator reaches the post despite the fast running flow of information. Thus, the escapism that the ephemeral spectacle entails conjugates picturesque conventions. Such a conception of the picturesque enables the spectator to be settled across the incommensurable wilderness of high-speed society. Landscape, as the primary subject matter of the picturesque aims for "the evacuation of verbal, narrative, or historical elements and the presentation of an image designed for transcendental consciousness" (Mitchell 2002, viii). According to Kant, aesthetic contemplation proceeds from a disinterested engagement and therefore permits universal communication (Kant 2008). Picturesque visuality is contemplative in this sense because it allows space for the spectator to look beyond the self.

Social media's limiting posts to a temporary lifespan affects the user's experience in formulating the posts as well as how they can be seen and how they gain meaning. Social interactions on Instagram stories are mediated through the visualization and interaction with the story shared by the users. Any view of an Instagram story automatically sends a notification to the story's producer that the story has been viewed, while such a connection does not leave a trace. For instance, when a user visualizes ephemeral content, he may react or comment on the publication as well as send a message to the owner of the shared content. In comparison to the bombardment of visuals in the newsfeed, the mass appreciation of temporal stories increases the visibility of the posts. In a case study of Instagram stories, for $83 \%$ 
of participants the temporariness of this shared content is a motivational factor once the stories change frequently (de Souza Silva et al. 2018). As such, users of Instagram stories affirmed that they like to see who will express a reaction to their posts before the expiration time (de Souza Silva et al. 2018, 44). Ephemeral posts create attention and hence awareness in visual abundance while creating a sense of urgency for users to check the platform in hopes of not missing any content (Khalil and Najaf 2019, 164). In this sense, temporal media is not historical, yet more connective thanks to its authenticity claim.

The authenticity claim firstly derives from the fact that the preparation of ephemeral posts demands less planning, staging and editing, which is different from permanent content. Thus, the use of temporal stories consumes less time while maintaining engagement between the participant and the audience (Chen and Cheung 2019; Khalil and Najaf 2019). Users are more confident sharing content that does not appear in the main content feed, while they are experiencing a feeling of authenticity (Xu et al. 2016; Bayer et al. 2016). Social media participants report that they are using this platform mainly to share their great moments with friends and to see how these friends react to this shared content (de Souza Silva et al. 2018). In claiming authenticity, these posts allow the audience to experience particular yet not specifically grandiose moments of life. Such experiences are able to claim authenticity precisely because perishable posts stand against the assumed historical register that "document[s] series of events to suggest that something occurred rather than nothing at all" (Petro 1993, 78). The ephemerality of the image offers an "aesthetics of fluidity and impermanence: the format of the platform constantly changes, contributions do not last, and identities created through the app are a 'liquid self'" (Verstraete 2016, 108). These stories mark a break with archival personal story-making while promoting a sensible yet intelligible spectacle of a glimpse. More importantly, in contrast to the brief duration of the spectacle, ephemeral profile stories reflect a pause between the self and its experience. They mediate a momentary yet contemplative visual to the viewers. The temporal feature of these stories constitute a glimpse of an alternative self-narrative that is generally defined as a lifespan process beginning in early childhood and extending to old age, as well as a process that holds societal expectations. Unlike a series of fixed categories adding up to a proper social media profile, such selfies exhibit the presence of the present experience of self-contemplation, a glimpse of thought, and not an eventful practice in visual mode. Thus, "being in the scene" pathos eventually communicates having distance and being conscious of how social reality is constructed (Manovich 2016, 136). In comparison to Instagram stories, another 
perishable media post, livestreaming, does not constitute a pause in viewing. Livestreaming does not reflect such a picturesque stance because it emphasizes "real time" and the "nowness" of the spectacle. Consequently, livestreaming does not form an alternative temporality to high-speed society because it prioritizes active spectatorship rather than a contemplative understanding and deliberation. The priority of "real time" does not facilitate an experience of the "presence of present moment" but is based on tracking with the newest visual appearing on the flow. Therefore, livestreaming as a form of interaction design devaluates contemplation of the visual.

The specific visual spectacle of temporal stories contradicts the fragmented everydayness conditioned by the obligations of high-speed immediacy. The picturesque view similarly mediated the pastoral refuge at the edge of the city line. Thus, authenticity derives from recognition of time and the finding of one's Being-there in these posts. Selfie image is a matter of "self-manifestation, selfdesign and self-positioning in the aesthetic field;" therefore, it obviously fails to invite disinterested engagement, yet it appears to be our contemporary form of self-contemplation (Groys 2011, 34-38). These temporal posts generically present a point of view shot of a momentary part of user's everyday life. In fact, viewers begin watching stories by pushing on the story producer's image which runs in a list along the top part of user's newsfeed without any means of pre-viewing the visual posted. Followers are limited to watching the Instagram Story, skipping it or commenting on it in order to start a private conversation. The entire mobile screen as frame secures the observer's safety in another place for surveillance. While posting Instagram stories, users can add textual captions such as time, location, temperature and emoji as well as a thematic hashtag [Fig. 1]. ${ }^{1}$ The story delivers "context," in other words, the information about the surrounding situation, with an emphasis on encompassing features (Bayer et al. 2016, 4). Such Being-there visuals reclaim a break in the flow of the high-speed connective mode that engages not only the post producer but also the indeterminate viewer among followers. Therefore, it mediates thought of the self in a moment of time in a disinterested manner. Here, mediated voyeurism is not simply based on an imitation of reality. The visual of the Instagram story is selected by the producer to be commented on and then shared for public view and communication. The visual image, among others, can be of protagonist's view fitted to be perceived by the producer and would-be-spectator. For instance, the exemplary visuals in Figure 1 reflect such self-contemplative features not only because they refer to stillness without eventful progression but

1 All of Instagram stories seen in the figure are public stories with public view. 
also because they project its producer's thinking and hence the contemplative mode of its own momentary narrative.

Fixed media, such as painting, sculpture, and photography are considered to reflect a presence because they typically prioritize depiction rather than the recreation of movement (Westgeest 2015). Thus, the viewer reflects an experience of the still as a pause in the flow. Thereby, in addition to the urgency of the audience to see otherwise missed content, the short but un-interrupted viewing time offers an extended spectacle. In that sense, such temporality remedies the poor condition of the attention span. Therefore, image production can take a break and image contemplation can proceed. In the case of Instagram moving stories, the movement of the visual persists. However, the ephemeral feature of posts as much as the limited duration of the story only offer a glimpse of movement without progress or event. Although the moving stories of temporal posts exhibit the display of a succession of images without a pause, the brief lifespan holds the attention of the viewer through redoubling the act of watching. Such stories do not inform the viewer about the background context. Thus, a glimpse of movement puts demands on the viewer's imagination beyond what the visual perception permits. Goswamy (2014) has defined ruminative viewing for Indian paintings with a cyclical time reference as such, "each work of art comes out of a particular time and always, consciously or unconsciously, references it" (Goswamy 2014, section IV), and claims that each culture has its own specific time reference. The self-focus inherent in ruminative thinking is assumed to take on peculiarity in the details of the visual story post among the vast digital culture of social media. The temporal and spatial isolation of the visual from the broader narrative of social media does not guarantee the consumption of these visual details. Yet, it attests to the importance of the post and the act of seeing rather than looking. Indeed, contemplation derives not only from the act of noticing the image but also from the process of deliberation. Atkinson's study of comics states that both the quality of the image and its presentation render visual thought possible in the way of making the eye continue to be intrigued (Atkinson 2012, 67). Similar to the presentation of comics' frames to the viewer, the presentation of the Instagram moving story presents a contemplative pause due to early interruption of the movement. For instance, one of the Instagram moving stories seen in Figure 2 captures a taxi trip, the other a fun game among friends. The design of these Stories features a sudden start and end, which aims to set in motion the spectator's participation through the viewer's imagination of the story's complete setting. The faint memory of the story watched is envisaged to leave a trace on mind. The viewer combines this trace in their imagination with the elements of 
this visual glimpse. Thus, the Instagram moving story drops into the stillness by the sudden cut which arrives at the end of 15 seconds.

The time dimension of the Instagram stories' experience design imposes an awareness of time. The culture of social media has a specific sense of viewing culture. For instance, Instagram stories differ from the archival mode of social media newsfeeds in the sense of its contemplative pause for exhibiting and viewing visuals. Newsfeed scrolling practice differs from stories moving forward and backward through leftright direction. The viewers have more control on their own time of appreciation on the newsfeed as they scroll down. Yet the posts of still image and moving images do not have any duration unless they attract the spectator's attention. Laura Mulvey argued that the eruption of the still is a radical break on new technologies giving the spectator control of the viewing process (Mulvey 2007, 135). The spectator of social media assumingly owning full control of viewing newsfeed in Instagram just follows the rule of speed and respectively performs the habit of scrolling down the visual news mostly without "time to stop, look and think" (Mulvey 2007, 135). The reduction of movement for the viewer facilitates contemplation, thus, stories whether still or moving images of 15 seconds exhibit no change or movement without event. Without the skip move, the viewer has no choice but to wait until the Instagram story ends and watch how the story proceeds. Generically, the spectator seems to control newsfeed, although such control does not attract the spectator's attention due to the flow of ubiquitous social media production. In that sense, contradictory to what Mulvey envisions for digital technologies to enable the viewer's full control for thoughtful contemplation, the high-speed temporality of the digital public does not permit such agency unless design intervenes. Instagram's ephemeral stories encompass the screen for a short time, without an easy get out. The contemplation time has been co-directed by the viewer while she/he selects the story that pops up through the images posted by the account holder. But more importantly, this viewing time of Instagram stories has been decided collectively namely by both post sender and receiver. Thereby, such semi-loss of control marks a radical break on the spectator's technologies of social media practice.

In this vein, ephemeral stories have drastically transformed social media viewing culture because they do not only dictate the time needed to view them but also the time to contemplate visuals. Spectators of stories tend to be accidental witnesses of one's self-contemplation, whose duration is not in their control. The view of selfcontemplation is picturesque in the sense that "the standard picturesque landscape is pleasing because it typically places the observer in a protected, shaded spot (a 'refuge')" (Mitchell 2002, 16). The spectator's passive interaction secures their contemplation in 
some sense. Generically, social media does not permit the user viewer/view producer "to add to the image" (Bellour 2007, 123). However, features such as Instagram stories can work as a stop in the high-speed immediacy reign. Thus, the temporality of these posts presents a shift in the nature of spectatorship in social media.

\section{The Picturesque Online}

The self-image construction invites both the post producer and the viewer to view the self. Here, picturesque aesthetics consists of the temporal contrast of the ephemeral visual post vis-à-vis everyday life in a high-speed society. Boredom coincides with "both too much and too little, sensory overload and sensory deprivation, anxieties of excess as well as anxieties of loss" (Petro 1993, 81). Once the graphic artists had to arouse minds held by information overload and boredom (Lyotard 1999, 40), now any active participant of social media must also stimulate others in order to be interacted with. Thus, the picturesque landscape locating the self in nature mediates the looking at the self there holding on yet fading along a digital layout of high-speed communication.

The interaction and user experience design of temporal stories differs from other social media features in the sense that the duration of contemplation is not completely directed by the viewer. In a way, a 15-second Instagram story, if it is watched from the beginning to the end, is much longer than a short look at a given image in the newsfeed or on livestreaming. The authenticity of such ephemeral posts coincides with its focus on the momentary gaze of one's experience. Yet such a view illustrates a glimpse of thought rather than the sensible representation of reality as the viewer witnesses the poster's contemplative gaze directed towards the self. Thereby, it offers an intelligible mode for the viewer by granting time that is isolated from the speedy flow of instantaneous messages. Thus, it is not only the post's short lifespan of 24 hours but also the limited duration of 15 seconds which extends visual appreciation and hence adds value to the image.

This escape from high-speed society can eventually become insecure. Here, the picturesque refuge of social media consists of virtues of such perpetual contrast that they can eventually provoke a sensory response back to the bored viewer. In that sense, the picturesque frame renders the sublime a loss of self in the high-speed immediacy which is compromised with the temporal system. As Robinson argues, the picturesque is a position of the one that "packs a lunch and good book to set out for just a day of ease on a hillside looking back to the city" (Robinson 1988, 75). Thus, "the picturesque is subject to the dominant power beyond the hill" (Robinson 
1988, 75). The picturesque can even be illusory for Robinson (1988) because it enables such a break to stimulate and energize the subject. For instance, the visual experience of Instagram stories can be relieving for users otherwise suffering from profound boredom and hence remedying indifference regarding what the highspeed society offers. From this angle, the picturesque on social media can distract its users through disorienting them to societal temporality. Such disorientation can serve to naturalize the very same high-speed society.

The temporal contrast that the visuality of ephemeral social media provokes is stimulating. Such need for a contemplative pause as it relates to the online spectacle illustrates the symptoms of our high-speed society's inconsumable presence. The interaction design centred on user experience of Instagram stories offers time to the viewer to contemplate. The spectator not completely controlling the viewing duration can involve themselves in the experience of various temporalities that exist beyond one's own. Indeed, the longevity of the popularity of such temporal spectacle, namely stories, in social media depends on its embodiment of the picturesque view. However, the increase in ephemeral story features in every social media platform and in the number of its users would eventually decrease the stimulation potential of such features. Until this point, the ephemeral media of self-contemplation stand still against the bombardment of social media posts and offer a picturesque refuge.

\section{References}

Alhabash, Saleem and Mengyan Ma. 2017. A Tale of Four Platforms: Motivations and Uses of Facebook, Twitter, Instagram, and Snapchat Among College Students? Social Media + Society vol. 3, no. 1: 1-13.

Atkinson, Paul. 2012. Why Pause?: The Fine Line between Reading and Contemplation. Studies in Comics vol. 3, no. 1: 63-81.

Bayer, Joseph B., Nicole B. Ellison, Sarita Y. Schoenebeck and Emily B. Falk. 2016. Sharing the Small Moments: Ephemeral Social Interaction on Snapchat. Information, Communication \& Society vol. 19, no. 7: 956-977.

Bellour, Raymond. 2007. The Pensive Spectator. In The Cinematic, ed. David Campany, 119-123. Cambridge, MA: The MIT Press.

Carr, Caleb T. and Rebecca A. Hayes. 2015. Social Media: Defining, Developing, and Divining. Atlantic Journal of Communication vol. 23, no. 1: 46-65.

Chen, Kuan-Ju and Hoi Ling Cheung. 2019. Unlocking the Power of Ephemeral Content: The Roles of Motivations, Gratification, Need for Closure, and Engagement. Computers in Human Behavior vol. 97 (August): 67-74. 
Clement, J. 2020a. Instagram Stories Daily Active Users 2019. Statista. https://www. statista.com/statistics/730315/instagram-stories-dau/. Last accessed 08. 01. 2020.

Clement, J. 2020b. Global Social Media Ranking 2019. Statista. https://www.statista. com/statistics/272014/global-social-networks-ranked-by-number-of-users/. Last accessed 01. 02. 2020.

Duin, J. van. 2018. Instagram Stories: Giving Shape to a New Ephemeral Photographic Experience. Masters' thesis. http://dspace.library.uu.nl/handle/1874/369185. Last accessed 28. 01. 2021.

Fallman, Daniel. 2008. The Interaction Design Research Triangle of Design Practice, Design Studies, and Design Exploration. Design Issues vol. 24, no. 3: 4-18.

Goswamy, B. N. 2014. The Spirit of Indian Painting: Close Encounters with 100 Great Works 1100-1900. New Delhi: Penguin.

Groys, Boris. 2011. Going Public. Berlin - New York: Sternberg Press.

Hammer, Espen. 2004. Being Bored: Heidegger on Patience and Melancholy. British Journal for the History of Philosophy vol. 12, no. 2: 277-295.

Hand, Martin. 2016. \#Boredom: Technology, Acceleration, and Connected Presence in the Social Media Age. In Boredom Studies Reader: Frameworks and Perspectives, ed. Michael E. Gardiner and Julian Jason Haladyn, 115-130. Oxfordshire: Taylor \& Francis.

Heidegger, Martin. 1962. Being and Time. New York, NY: Harper and Row Publishers. Heidegger, Martin. 1996. The Fundamental Concepts of Metaphysics: World, Finitude, Solitude. Bloomington: Indiana University Press.

Helsinger, Elizabeth. 2002. Turner and the Representation of England. In Landscape and Power, ed. W. J. T. Mitchell, 103-126. Chicago: University of Chicago Press.

Illing, Sean. 2019. Bored and Lonely? Blame Your Phone. Vox. https://www.vox. com/recode/2019/5/2/18510958/social-media-addiction-boredom-lonelinesssociety-technology-smart-phones. Last accessed 02. 05. 2019.

Javed, Sabih. 2019. 7 Tips for Your Instagram Story Size. Lumen5 Learning Center (blog). https://lumen5.com/learn/instagram-story-size/. Last accessed 21. 05. 2019.

Jurgenson, Nathan. 2013a. Temporary Social Media. https://newsroom.snap.com/ temporary-social-media/. Last accessed 06. 03. 2021.

Jurgenson, Nathan. 2013b. Pics and It Didn't Happen. The New Inquiry (blog). February 7. https://thenewinquiry.com/pics-and-it-didnt-happen/. Last accessed 03. 02. 2020.

Kant, Immanuel. 2008 [1790]. Critique of Judgement. Revised edition. Oxford New York: Oxford University Press. 
Khalil, Israfilzade, and Babayev Najaf. 2019. Millennials versus Non-Millennials: The Context of Engagement Levels on Instagram Stories. In Challenges of Globalization in Economics and Business, The 4th International Scientific Conference, 163-170. Tbilisi, Georgia.

Kofoed, Jette and Malene Charlotte Larsen. 2016. A Snap of Intimacy: Photo-Sharing Practices among Young People on Social Media. First Monday vol. 21, no. 11. https://firstmonday.org/article/view/6905/5648. Last accessed 06. 03. 2021.

Kracauer, Siegfried. 1995. The Mass Ornament: Weimar Essays. Cambridge, MA: Harvard University Press.

Kress, Gunther and Theo van Leeuwen. 1996. Reading Images: The Grammar of Visual Design. Abingdon: Routledge.

Ladly, Martha. 2007. Being There: Heidegger and the Phenomenon of Presence in Telematic Performance. International Journal of Performance Arts and Digital Media vol. 3, no. 2-3: 139-150.

Lee, Eunji, Jung-Ah Lee, Jang Ho Moon and Yongjun Sung. 2015. Pictures Speak Louder than Words: Motivations for Using Instagram. Cyberpsychology, Behavior, and Social Networking vol. 18, no. 9: 552-556.

Lyotard, Jean-François. 1999. Postmodern Fables. Minneapolis, MN: University of Minnesota Press.

Manovich, Lev. 2016. Instagram and Contemporary Image. Cultural Analytics Lab. http://manovich.net/index.php/projects/instagram-and-contemporary-image. Last accessed 15. 11. 2020.

Misek, Richard. 2010. Dead Time: Cinema, Heidegger, and Boredom. Continuum vol. 24, no. 5: 777-785.

Mitchell, W. J. T., ed. 2002. Landscape and Power. Chicago, Illinois: University of Chicago Press.

Mulvey, Laura. 2007. Stillness in the Moving Image. In The Cinematic, ed. David Campany, 134-140. Cambridge, MA: The MIT Press.

Petro, Patrice. 1993. After Shock / Between Boredom and History. Discourse vol. 16, no. 2: 77-92.

Preece, Jennifer, Helen Sharp and Yvonne Rogers. 2015. Interaction Design: Beyond Human-Computer Interaction. Hoboken, New Jersey: John Wiley \& Sons.

Reckwitz, Andreas. 2002. Toward a Theory of Social Practices: A Development in Culturalist Theorizing. European Journal of Social Theory vol. 5, no. 2: 243-263. 
Robinson, Sidney. 1988. The Picturesque: Sinister Dishevelment. In Restructuring Architectural Theory, ed. Marco Diani and Catherine Ingraham, 74-79. Evanston, Illinois: Northwestern University Press.

Rosa, Hartmut and William Scheuerman. 2009. High-Speed Society: Social Acceleration, Power, and Modernity. University Park, PA: Penn State University Press.

Sheldon, Pavica. 2008. The Relationship Between Unwillingness-to-Communicate and Students' Facebook Use. Journal of Media Psychology Theories Methods and Applications vol. 20, no. 2: 67-75.

Sheldon, Pavica and Katherine Bryant. 2016. Instagram: Motives for Its Use and Relationship to Narcissism and Contextual Age. Computers in Human Behavior vol. 58: 89-97.

Sheldon, Pavica, Philipp A. Rauschnabel, Mary Grace Antony and Sandra Car. 2017. A Cross-Cultural Comparison of Croatian and American Social Network Sites: Exploring Cultural Differences in Motives for Instagram Use. Computers in Human Behavior vol. 75: 643-651.

Smith, Aaron. 2015. A 'Week in the Life' Analysis of Smartphone Users. U.S. Smartphone Use in 2015. Pew Research Center. https://www.pewresearch.org/ internet/2015/04/01/us-smartphone-use-in-2015/. Last accessed 06. 03. 2021.

Souza Silva, Thiago Alexandre de, Glívia Angélica Rodrigues Barbosa, Ismael Santana Silva and Flavio Roberto dos Santos Coutinho. 2018. Ephemeral Narrative Systems' Sociability Mediated by User Experience: A Case Study of Instagram Stories. IADIS International Journal on WWW/Internet vol. 16, no. 2: $34-49$.

Valentini, Chiara, Stefania Romenti, Grazia Murtarelli and Marta Pizzetti. 2018. Digital Visual Engagement: Influencing Purchase Intentions on Instagram. Journal of Communication Management vol. 22, no. 4: 362-381.

Verstraete, Ginette. 2016. It's about Time. Disappearing Images and Stories in Snapchat. Image \& Narrative vol. 17, no. 4: 104-113.

Virilio, Paul. 2005. The Information Bomb. Brooklyn, New York: Verso Books. Virilio, Paul. 2012. The Administration of Fear. Los Angeles, CA: Semiotext.

Westgeest, Helen. 2015. Video Art Theory: A Comparative Approach. Malden, MA: Wiley-Blackwell. 
Whiting, Anita and David Williams. 2013. Why People Use Social Media: A Uses and Gratifications Approach. Qualitative Market Research: An International Journal vol. 16, no. 4: 362-369.

$\mathrm{Xu}$, Bin, Pamara Chang, Christopher L Welker, Natalya N. Bazarova and Dan Cosley. 2016. Automatic Archiving versus Default Deletion: What Snapchat Tells Us About Ephemerality in Design. In Proceedings of the 19th ACM Conference on Computer-Supported Cooperative Work \& Social Computing - CSCW '16, 16601673. San Francisco, California: ACM Press.

Zimmerman, John, Jodi Forlizzi and Shelley Evenson. 2007. Research through Design as a Method for Interaction Design Research in HCI. In Proceedings of the SIGCHI Conference on Human Factors in Computing Systems - CHI '07, 493-502. San Jose, California: ACM Press.

\section{List of Figures}

Figure 1. Captions of Instagram stories.
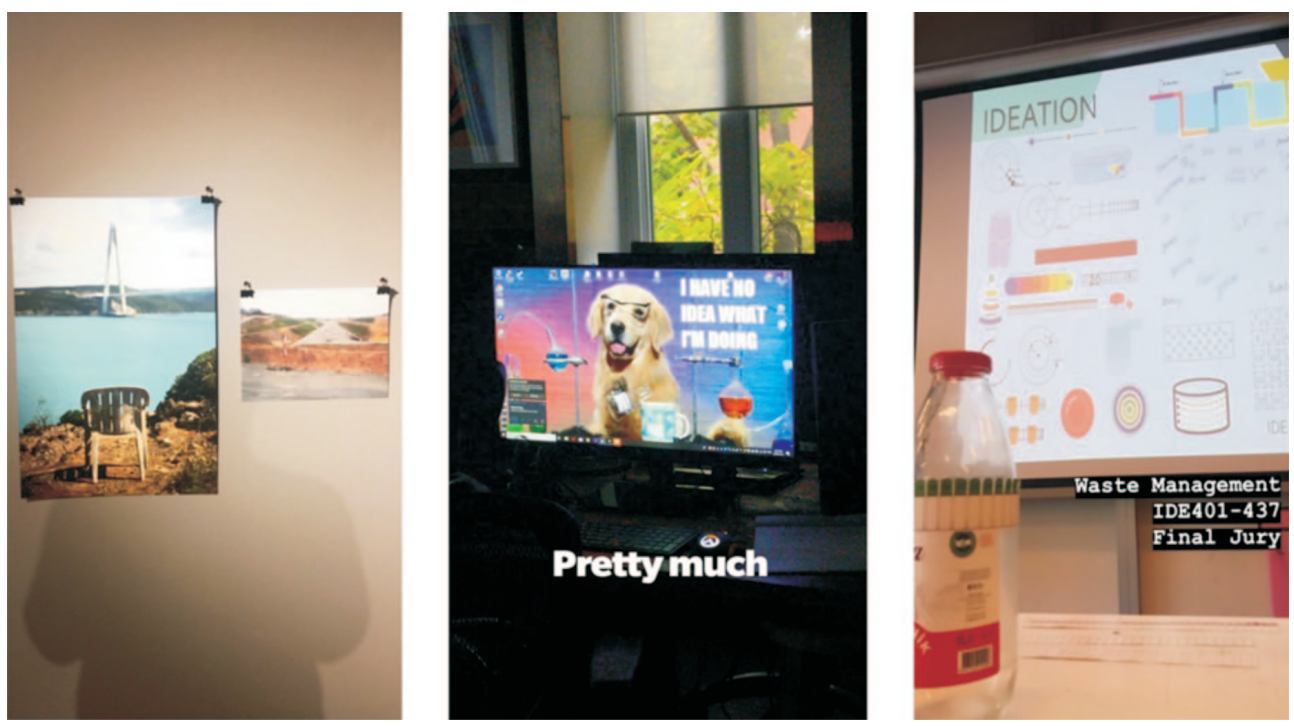
Figure 2. Captions of randomly selected Instagram moving stories.

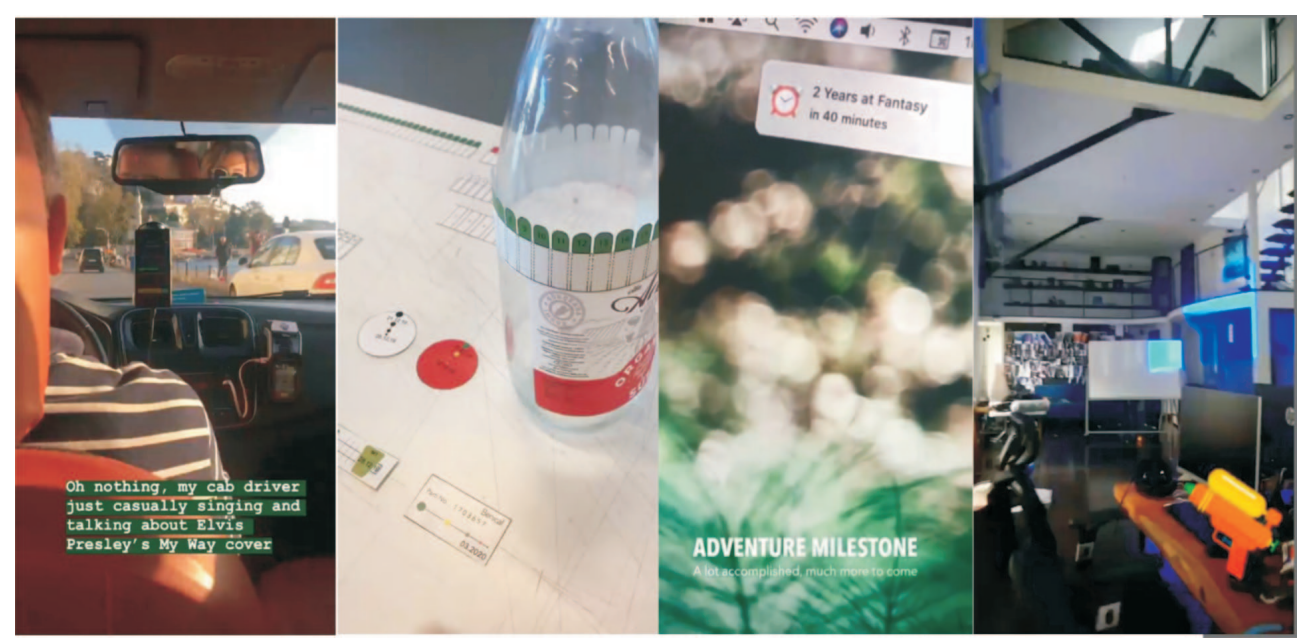

